audit and how they propose to use them in a practical programme. The cost of recruiting and training audit analysts will certainly be less than the cost in opportunity of diverting clinicians from clinical practice. There is also therefore an economic argument for selecting criterion based audit rather than more traditional methods.
1 Royal College of General Practitioners. What sort of doctor? London: RCGP, 1985.

2 Coles C. Self assessment and medical audit: an educational approach. Br Med $\mathcal{F}$ 1989:299:807:8.

3 Donabedian A. Advantages and limitations of explicit criteria for assessing the quality of health care. Milbank Memorial Fund Quarterly 1981;59:99-105.

4 Jessee WF. Criterion based screening. Identifying health care quality problems: $a$ practical manual for PSROs and hospitals. Chapel Hill: University of North Carolina School of Public Health, 1982.

\section{Random review of hospital patient records}

\section{A Heath}

In 1978 the departments of medicine and clinical pharmacology of Birmingham University instituted a regular weekly medical audit meeting. Details of the meetings and the effects of audit have been previously described. ${ }^{12}$ Basically, notes were chosen randomly from inpatient admissions once the final discharge summary had been completed. The notes of one consultant firm were then reviewed by another consultant firm, which commented on various points, including the quality of the notes at admission and follow up, appropriateness of investigations and drug treatment, speed of producing discharge summaries and their content, and evidence of communication among staff, patient, and general practitioner. These meetings were held regularly and successfully for many years. During the hour devoted to audit each week two sets of notes were analysed for each consultant firm and hence only a fraction of the total number of inpatients managed by the firm were reviewed. This style of audit has now been introduced throughout all medical firms in this hospital, primarily as a result of the decision of the Royal College of Physicians of London to make audit an essential feature of junior doctor training.

In this article I will discuss the advantages and disadvantages of this form of audit as practised by the original four consultant firms and now by 14 consultant firms. The views are mine and do not necessarily reflect those of my colleagues.

The advantages of the orignial audit were that, as practised, it was simple to do and entailed little administrative time, and no computers or additional staff were required as the secretaries merely choose at random two sets of notes for which a discharge summary had recently been completed. As only four consultant firms were concerned notes from each firm were analysed at each meeting so that all staff present were involved in the meeting. This also meant that an appreciable proportion of the work of each firm was audited, perhaps $10-20 \%$ of all admissions.

The disadvantages were that most notes were not audited, so that major mistakes could easily be missed; no attempt was made to audit outpatient practice; and by auditing after the patients had left hospital, it was too late to alter their management. The process did not entail other groups involved in patient managementfor example, nursing staff, general practitioners, etc. In fact, nursing staff attended some of the early meetings, but it proved impossible to arrange for them to leave the ward regularly to attend.

\section{Lessons and achievements}

Despite these disadvantages several important

Department of Medicine, Queen Elizabeth Hospital, Birmingham B15 2TH

D A Heath, FRCP, consultant physician

BrMed f 1990;300:651-2 had made them. For instance, a patient of mine was prescribed spironolactone at a time when the serum potassium concentration was $>6 \mathrm{mmol} / \mathrm{l}$. How could this happen? It had to mean that prescribing at times took place without reference to investigations. The identification of the mistake allowed the ward practice to be reviewed. Although in this example the prescription was unequivocally wrong, most discussions usually centred around the appropriateness of certain investigations or treatments, when there is often no absolute answer. This disclosed that much of our medical practice was often based on habit rather than medical facts. Often no unified conclusion emerged-for instance, there is no one correct way of investigating an elderly patient with an iron deficiency anaemia or a patient with a swollen leg. Almost imperceptibly, however, after several discussions of similar cases, policies started to change and become more uniform.

\section{... medical practice was often based on habit rather than medical facts.}

Initially, reporting of the illness and subsequent progress was poor; information on what had been said to patients regarding illness and progress in the inpatient notes and correspondence was almost non-existent. All these deficiencies improved immediately audit was instituted. Although it was possible to measure and show the benefit of audit on reporting in the notes and discharge summaries, ${ }^{2}$ it was more difficult to do so for investigations and treatment and impossible for patient morbidity and mortality. The failure to show an effect of audit on investigations was at first sight disappointing but, on reflection, expected. The average general medical admission is an emergency admission with a condition that does not require extensive investigation and often settles rapidly - for example, asthma or heart failure-or, if not, requires long term management rather than prolonged investigation-for example, a dense stroke. To show an effect of audit on investigations I suggest that the elective investigation of a specific problem would need to be chosen. There are, however, few specific conditions regularly investigated by most doctors, making local comparisons difficult. The failure of this form of audit to have any demonstrable effect on outcome, has been leapt upon by some as an indication of the lack of benefit of audit and used to resist its introduction. It would be amazing, however, if this form of audit could be shown to affect, say, mortality in general medicine; you have only to think of the size of trials required to show an effect of treatment on survival after a myocardial infarction. No one city, let alone one hospital or one consultant, could expect to show significant effects of management on and foremost, it became clear that audit coul in a friendly, non-confrontational manr a form that was enjoyed by all who participated. Major mistakes were, in fact, uncommon and, when identified, were usually incomprehensible even to those who 
morbidity or mortality after, say, a haematemesis, asthma attack, stroke, or overdose unless gross medical mismanagement was regularly taking place.

Once the meetings were well established and the problems of reporting dealt with, a problem of repetitiveness occasionally arose. One final point to emerge was that for audit to continue to be valuable it had to be supported by consultants who believed in its benefit and who were regular attenders.

\section{Re-emergence of hospital audit}

The directive of the royal college that medical audit must be seen to be taking place in hospitals training junior staff has led to the re-emergence of audit in this hospital, this time involving all physicians, both general and specialised. An identical format is being used, but the participation of all types of physicians has brought advantages and disadvantages. The disadvantages are the difficulty of finding a suitable time for all consultant teams to meet; also the increased participation of other firms automatically reduces the proportion of case notes examined in each firm to a low value. Currently, we meet each week and two firms are audited, meaning that any one firm will be audited every two months. The disadvantages have to be balanced by the participation of firms who previously did not conduct audit

\section{Most weeks at least one important problem that warrants attention is unearthed.}

and by the ability to have specialist opinions on most topics.

Despite initial protestations the meetings have been well attended and some initial doubters have admitted to their value. Particularly encouraging has been the recognition by some of the consultants that now audit has to be done it is in our interest to try to do it properly. Most weeks it is considered that at least one important problem that warrants attention is unearthed. Of particular interest to me is the discovery of common practices that are unknown by most of the consultants despite the fact that they were occurring on their firms - a recent example being that most patients admitted to the hospital with a chest infection were having blood cultures performed. Now that the audit process has been accepted by the physicians it is clear that the format of future meetings will need to be changed. A mechanism needs to be set up to ensure that queries or problems identified at the meetings are properly researched or followed up. To this end it is planned to have nominated consultants to do this and to report back to subsequent meetings. Several of the laboratories and support services have asked to be present at meetings to discuss the way they are being used or misused by the clinicians. They can be incorporated into the rota, and this will help to maintain the interest of the meetings.

Although I have had it pointed out that the random case review seems to be directed almost exclusively at looking for mistakes and does not seem to want to record how well we practise medicine, it seems to me to be far more important to become aware of the occasions that we perform inefficiently or badly rather than applaud those when we do well, which we inevitably recognise anyway. Our current method is guaranteed to miss most problems. However, the principle of audit is to try to make doctors think constantly about why they are doing things and may in itself lead to a reduction in errors. Despite this it would be a major advantage to identify cases in which errors are more likely to have occurred. Concentrating only on deaths is unlikely to be worthwhile as most are unavoidable. Certain categories might be identified for automatic review - for example, all deaths under 40 , deaths after gastrointestinal bleeding, those after asthma, etc. Another strategy is the process of severity adjusted analysis.

\section{Selecting cases for audit}

The length of time that a patient stays in hospital depends on various factors; primarily, it depends on the illness and is also influenced by any other coexistent diseases, so that a patient with chronic liver disease who has gastrointestinal bleeding is likely to need a longer stay in hospital than one without. Average times that any patient might be expected to stay in hospital may be calculated for a series of primary conditions, and these can be modified for the confounding effects of age and other classified disorders present. The practice of a hospital can then be examined and patients identified who stayed in hospital for a longer than expected period. Such patients will have stayed in hospital longer for various reasons, including complications of the disease (which may or may not have been preventable) personal policy of the management team, unavailability or poor use of facilities to permit efficient investigation of a problem, etc. These patients, if they can be selected, comprise a group that merits examination by audit and that is more likely to disclose problems that require identification than the random selection of patients who probably have had a short, uneventful stay in hospital. The surveillance of proper reporting can continue just as well on this selected group of patients.

Severity adjusted analysis is totally dependent on the proper and complete identification of the medical conditions present. This information at present should be collected to provide Körner data. Very little effort is made to check such data, and all the evidence is that it is often inaccurate or incomplete. Yet this information is currently being used to calculate the allocation of funds to different hospitals. If the data can be improved then evaluating severity adjusted analysis to see whether it really selects a group of patients who are more relevant for auditing should be possible. I hope that such a scheme at this hospital may be evaluated in the near future. This discussion of cases that have not progressed satisfactorily by specialists from many medical disciplines should permit the emergence of better practices. Such discussions would have a major educational role for both junior and senior doctors.

The decision of the college to expect audit to take place in all hospitals has given a massive impetus to developing proper auditing. I hope that its widespread introduction will have convinced non-believers that its primary use is to try to ensure a high level of patient care. It is not a weapon to save money or to punish doctors, and, used sensibly, it may help our medical practice rather than hinder it. Techniques of auditing are still in their infancy, and with wider experience different and better methods will be found; rather than resist or mock the present system it behoves the medical profession to support the development of audit and to improve patient care.

1 Heath DA, Hoffenberg R, Bishop JM, Kendall MJ, Wade OL. Medical audit $\exists R$ Coll Physicians Lond 1980;14:200-1.

2 Heath DA. Medical audit in general medicine. $f R$ Coll Physicians Lond 1981;15:197-9. 